\title{
MODERNIDAD CAPITALISTA Y VALOR DE USO EN BOLÍVAR ECHEVERRÍA
}

\section{CAPITALIST MODERNITY AND USE-VALUE IN BOLÍVAR ECHEVERRÍA}

\section{Moisés Martínez Gutiérrez ${ }^{1}$}

\begin{abstract}
Resumen
La problematización del valor de uso es conducida por Marx a partir de su subordinación al valor de cambio, ya que para el modo de producción capitalista lo determinante es la realización del valor económico. En este sentido, Marx no desarrolla una crítica de la forma natural de la mercancía. Sin embargo, Echeverría retoma el valor de uso como parte importante de la crítica de la economía política, como expresión real y simbólica de una determinada sociedad. Desde esta idea, el foco de atención no estará en el objeto-mercancía, sino en el sujeto, cuyo código social da sentido a sus relaciones económicas. El presente artículo tiene por objetivo analizar conceptualmente la estructura argumentativa que propone Echeverría, comenzando por la caracterización que da Marx sobre la mercancía.
\end{abstract}

Palabras clave: forma abstracta; acuerdo; código social; forma concreta

\begin{abstract}
The problematization of use-value is considered by Marx from its subordination to exchange-value, since the realization of economic value is the determinant for the capitalist mode of production. In this respect, Marx does not give a critique of the natural form of commodity. However, Echeverría returns to use-value as an important part of the critique of political economy and as a real and symbolic expression of a given society.
\end{abstract}

Doi: http://dx.doi.org/10.15359/eys.22-51.2

Fecha de recepción: 17-12-2016. Fechas de reenvíos: 11-01-2017 / 06-02-2017 / 13-02-2017 / 27-02-2017. Fecha de aceptación: 01-03-2017. Fecha de publicación: 04-03-2017.

${ }^{1}$ Profesor adjunto en el Posgrado en Estudios Latinoamericanos de la Universidad Nacional Autónoma de México (UNAM), México. Realizó una estancia doctoral en el Centro de Investigaciones sobre América Latina y el Caribe (CIALC), de la UNAM. Correo electrónico: mmgu76@gmail.com

Moisés Martínez Gutiérrez

Revista Economía y Sociedad by Universidad Nacional is licensed under a CreativeCommons Reconocimiento-NoComercial- 
From this approach, focus will not be on the object-commodity but on the subject, whose social code gives meaning to economic relationships. This paper is aimed at conceptually analyzing Echeverría's theoretical argument beginning with Marx' characterization of commodity.

Keywords: abstract form; agreement; social code; concrete form

\section{Introducción}

Hoy, la crítica de la economía política debe tener en cuenta el análisis del valor de uso, considerándolo técnica y simbólicamente. Su carácter físico no explica por sí mismo la reproducción del sujeto social, la materialidad solamente adquiere sentido cuando el hombre proyecta en ella una forma propia, una forma de existencia cultural. Sin embargo, en el mundo de las mercancías lo que adquiere preeminencia es la modalidad del valor que la acompaña.

En su momento, Marx advirtió el absurdo de la economía moderna capitalista: la reproducción del ser humano estaba condicionada a la reproducción del valor. Con lo anterior, dejó entrever que, para el capitalista, los trabajos y productos concretos no son determinantes (hoy, el peso que tiene lo subjetivo en la determinación de la producción no modifica la esencia del capital); no obstante, no continuó más allá. Echeverría decide recuperar el tema del valor de uso y lo desarrolla desde un enfoque distinto: la producción-consumo de valores de uso es la producciónconsumo de significaciones, devolviéndole su forma de existencia cultural. De esta manera, el valor de uso deja de ser un simple rostro de la mercancía para convertirse en una expresión autónoma del sujeto social en un determinado momento.

La voluntad del sujeto social de dar un sentido a su objeto es un acto político (no económico). Por lo tanto, el sujeto productor es, primeramente, un sujeto político que da, a sí mismo y en reciprocidad, una forma determinada de su socialidad. Son los cambios al interior de la sociedad los que marcan el paso y definen la objetividad de lo material. Dicho lo anterior, este artículo tiene el propósito de analizar conceptualmente la estructura argumentativa que sobre el valor de uso propone Echeverría, con el propósito de detectar las principales diferencias y avances con respecto al papel que Marx le asigna a dicho valor en su discurso teórico crítico.

\section{La forma dual contradictoria}

La forma representativa del capitalismo es, básicamente, la forma dual contradictoria: la mercancía. Echeverría (1998a) menciona que Marx ve en ella la expresión de todas las contradicciones de la vida moderna capitalista. Dicha forma fue normalizada como el agente de la integración o unificación económica, vía intercambio; fenómeno moderno que Marx ya advertía en el siglo XIX, propio de una realidad social que puede ser caracterizada como:

2

Moisés Martínez Gutiérrez

Revista Economía y Sociedad by Universidad Nacional is licensed under a CreativeCommons Reconocimiento-NoComercial-

Compartirlgual 4.0 Internacional License.

Creado a partir de la obra en http://www.revistas.una.ac.cr/index.php/economia 
Materialismo político, es decir, el hecho de que en la vida social aparece una primacía de la política económica sobre otro tipo de políticas (...) puesto en otros términos, la primacía de la sociedad civil o burguesa (...) en que la sociedad funciona como una lucha de propietarios privados por defender cada uno los intereses de sus respectivas empresas económicas (...) lo otro, el aspecto más bien comunitario, cultural, de reproducción de la identidad colectiva, pasa a un segundo plano. (Echeverría,2010a, p.16)

La necesidad de desenvolvimiento autónomo de la forma dual contradictoria (necesidad marcada por la constitución abstracta y general de la mercancía) suponía un mecanismo capaz de regularse por sí mismo: el mercado de precios generalizado. En otras palabras, liberarse de cualquier determinismo de la naturaleza y de cualquier orden social tradicional: normas y prácticas de convivencia. La dialéctica mercado autorregulado/mercancía autónoma dio una imagen falaz: la totalidad intemporal. Un mito que, a decir de Polanyi (2009), desconoció al mercado regulado y focalizado, al comercio exterior complementario y a la diversidad de sistemas económicos anteriores al siglo XIX. Así pues, concretando la idea:

Desde el siglo XVI en adelante los mercados fueron numerosos e importantes. Bajo el sistema mercantil se convirtieron (...) en una preocupación principal de los gobiernos; pero aún no había señal del venidero control de los mercados sobre la sociedad humana. Por el contrario. La regulación y la regimentación fueron más estrictas que nunca; la idea misma del mercado autorregulador no se conocía. (Polanyi, 2009, p.84)

Ciertos acontecimientos fueron fundamentales en el desarrollo de aquella entidad económica que se separaba de la sociedad; por ejemplo, la unificación político-territorial de los Estados (la cual incentivó la constitución de un mercado interno o nacional), el crecimiento económico en las ciudades (movilizando la fuerza de trabajo), la creciente tecnificación de los procesos de producción (lo cual hizo que el trabajo fuera adquiriendo un perfil más homogéneo), etc.

Y así como la transición a un sistema democrático y a una política representativa entrañaba una inversión completa de la tendencia de la época, el cambio de los mercados regulados a los autorreguladores a fines del siglo XVIII representó una transformación completa en la estructura de la sociedad. (Polanyi, 2009, p.110)

Al liberarse la actividad económica del orden ethico antiguo, la forma dual contradictoria encuentra en el nuevo orden autorregulado el modo de su vinculación y realización. Dicho orden la vincula, socialmente, sobreponiendo lo cuantitativo a lo cualitativo; es decir, de acuerdo con su valor económico. Al revés, toda forma objetiva pre-moderna capitalista no puede ser considerada ni realizada por aquel orden. 
El fundamento ético del orden autorregulado exige (para su desarrollo adecuado) el cálculo óptimo y la eficiencia de los mecanismos que lo hacen posible; en otras palabras, se trata de una ética funcional (Hinkelammert, 2012). El bien común se transforma en el interés general, justificación ideológica del orden autorregulado.

El interés propio no es egoísta, sino es portador del interés general (...) Imponiéndose al mercado, todos son llevados por sus leyes a comportarse como servidores mutuos. La sociedad de mercado no es egoísta, sino es sociedad de servicio mutuo. (Hinkelammert, 2007, p. 90)

Se podría decir de otro modo: el servicio mutuo es la servidumbre al mercado. Esta forma de servicio capitalista, que tiene como escenario el mercado de oferta y demanda, hace de las relaciones humanas un simple intercambio de bienes y servicios, el cual es la forma en que se expresa el interés por el otro.

En el nuevo orden autorregulado se impuso entonces la lógica económica del beneficio calculado y, en este sentido, un modo de la existencia para las cosas: el valor y su revalorización capitalista (Echeverría, 2011). Lo creado por la naturaleza y trabajado por la acción del ser humano es así requerido; en su proceso formador el código social dominante (la parte sémica del objetomercancía) es dado por aquel modo de la existencia. Asimismo, ya que el telos del capitalismo se ciñe al valor y su reproducción, aparece otro mito, el mito de la trascendencia por el valor, ahí donde el hombre es el único capaz de generarlo y reproducirlo ilimitadamente.

La historia del proceso de valorización-enajenación capitalista, tratada teórica y críticamente por Marx, evidenció el conflicto entre la forma abstracta cuantitativa y la forma concreta cualitativa, dos formas antagónicas de la reproducción social. En dicho proceso operaba el espíritu de libre iniciativa con la ayuda de la técnica, la cual era gobernada por el principio de la producción del valor. De este modo se configura el ethos civilizatorio de la modernidad capitalista (Echeverría,2011).

Ahora bien, la Crítica de la Economía Política, efectuada por Marx en 1859 (ampliada posteriormente en su obra El Capital) tuvo como punto de partida el sistema de la economía burguesa, de acuerdo con un plan y un método ${ }^{2}$. Como parte de su sistema categorial (valor de cambio, equivalente general, plusvalía, etc.), el valor de uso es expuesto en calidad de soporte material de la mercancía.

\footnotetext{
${ }^{2}$ Rubel (2003) argumenta que el itinerario intelectual de Marx (el cual contemplaba, además de la crítica de la economía burguesa, las críticas sistemáticas a la moral, la política y el derecho) nunca fue completado. Razón para no considerar el pensamiento de Marx como un sistema cerrado, acabado, tal como los marxismos lo han querido ver.
}

4 
Si la estructuración del plan de trabajo de Marx (expuesta en el contenido capitular de El Capital) no consideró el desarrollo autónomo del valor de uso es porque su carácter práctico y determinado no explicaba, por sí mismo, las relaciones económicas en el nuevo orden autorregulado: "el valor de uso en esta indiferencia a la determinación económica formal, es decir, el valor de uso como tal, se halla fuera de la esfera de investigación de la economía política" (Marx, 1976, p. 22).

En las relaciones económicas de intercambio, las diferencias reales (las cualidades particulares) entre los productos se resuelven por su valor de cambio. Para Hinkelammert y Mora (2013), este hecho es más que una relación de valor, ya que, en el fondo, hay una abstracción de la función vital de los valores de uso, situación que Marx tiene presente en su crítica al capitalismo.

En consecuencia, el resultado de Marx es que cuando los productos del trabajo humano son medidos por el trabajo abstracto, esto es, cuando las relaciones humanas entre los productores se expresan como relaciones de valor entre los productos, y la acción económica humana se orienta por este indicador, se tiende a destruir las condiciones de posibilidad de reproducción de la vida humana. (Hinkelammert y Mora, 2013 , p. 331)

El valor de uso es, en el discurso teórico de Marx, una forma de existencia de la mercancía (la forma que la conciencia asume de la mercancía es una dualidad: la forma determinada y la forma indeterminada), la cual adquiere un sentido en el orden concreto de la sociedad capitalista. Al respecto, su cualidad singular tiene para el capitalista otra función distinta a la que le es propia. Con esto no se quiere decir que sus determinaciones como producto-técnico particular e ideal (Dussel, 1991) ya no interesan al capitalista (de lo contrario, sería imposible el intercambio y la realización del valor), pero ya no es condición suficiente.

Dussel (1991) sostiene que la crítica de Marx al capitalismo no está del lado del sujeto-consumidor sino del sujeto-productor, ahí donde la creación del valor es el comienzo del drama del trabajador. La preocupación por el contenido material de la mercancía o, desde otro ángulo de una misma relación dialéctica, la expectativa del consumo, real y simbólico, no explica por sí misma la problemática que quiere señalar Marx. Respecto al objeto cultural, Marx se limita a llevarlo por el mismo curso que el del valor de uso. Así lo explica Picó:

De ahí que para situar la cultura como producción material y espiritual de la humanidad en su lugar apropiado haya que vincularla a la estructura económica y social que predomina en cada momento histórico (...) tal como dice en el Prefacio (...). (Picó, 1999, p. 89) 
El tránsito del objeto práctico al objeto práctico mercantil, movimiento clave (El Capital, Capítulo I) para la comprensión del sistema capitalista (Echeverría, 2011), va a ser el comienzo de un cambio importante en la actividad económica del hombre. El valor de uso ya no tendrá manera de realizarse más que en su contrario, desdoblándose en la forma que expresa su valor relativo $y$, posteriormente, en la forma del valor en general: la forma dinero. Debe tenerse en cuenta que "la realización de una condición está directamente ligada a la realización de su opuesta" (Marx, $\underline{1976}$, p. 43).

La explicación que da Dussel de cómo Marx avanza metódicamente (analítica y dialécticamente) de lo simple a lo complejo aclara lo anteriormente comentado. En dicho movimiento la mercancía no puede quedarse como valor de uso. Este da paso a una nueva determinación que es general.

Puede observarse en ese "devenir" (el Übergang hegeliano) el pasar de una categoría a otra; del producto a la mercancía, de la mercancía al dinero. Son los dos primeros pasos de su discurso dialéctico (tanto en la Contribución de 1859 como en El capital del 67). Para Marx (...) el producto como la mercancía o el dinero son "formas" de existencia. (Dussel, 1991, p. 81)

La omnipresencia del valor de cambio (que crea el lazo social cósico) es ahora lo que comienza a dinamizar las relaciones económicas, las cuales, de acuerdo con Marx (1976), son protagonizadas por un individuo representativo (propietario y aislado) que aparece como puesto por la naturaleza. Inicialmente, son los propios productores los que hacen circular sus mercancías u objetos intercambiables.

En la sociedad mercantil, la forma natural del objeto está impedida de existir como realidad independiente y autónoma (...). El producto (...) debe esperar la autorización que viene de la mano oculta de la oferta y la demanda (...), autorización que consiste precisamente en la adjudicación de un valor de cambio al valor del objeto. (Echeverría,1998a, pp. 15-16)

En el horizonte del capitalismo temprano es claro el nuevo destino de la mercancía como objeto práctico, que continúa hasta el día de hoy. El objeto práctico movido por el valor de cambio (la manera de acceder a él) ha dejado atrás el principio fundamental del proceso de trabajo. Habría que recordarlo a través del propio Marx:

El proceso de trabajo (...) es la actividad racional encaminada a la producción de valores de uso, la asimilación de las materias naturales al servicio de las necesidades humanas, la condición general del intercambio de materias entre la naturaleza y el hombre, la condición natural eterna de la vida humana, y por tanto, independiente

6 
de las formas y modalidades de esta vida y común a todas las formas sociales por igual. (Marx, 1984, p. 136)

Ahora bien, el intercambio forma parte, en el planteamiento teórico-metodológico de Marx, de una totalidad: el sistema económico capitalista. Esto es señalado en la Introducción de Los Fundamentos de la Crítica de la Economía Política (1857), donde ve al sistema económico como una unidad, en la que cada elemento es mediación del otro.

Por consiguiente, llegamos a la conclusión de que la producción, la distribución, el cambio y el consumo no son idénticos, sino que cada una de estas categorías constituye un elemento de un todo y representa la diversidad en el seno de la unidad. (Marx, 1975, p. 36)

De esta manera, en el intercambio de mercancías, que es el "punto de partida de la argumentación global del Capital (...) de la Crítica de la Economía Política (...) como movimiento inicial, de un paso argumental (...)" (Echeverría, 2011, p. 567), el valor de uso cumple su finalidad en la totalidad: ser la mediación objetiva y subjetiva en el mundo de las mercancías. En un segundo momento, el valor, por sí mismo, toma el lugar del objeto práctico mercantil.

En cuanto tal, como objeto práctico de la sociedad, la mercancía es, de una primera perspectiva, una porción de materia concretamente útil o con valor de uso: un bien. $Y$ no solamente un bien en general (...) sino un bien específico o elemento que pertenece diferencialmente a la totalidad sistemática de lo que es efectivamente bueno o favorable para satisfacer las necesidades de consumo -en unos casos final o disfrutante, en otros intermedio o productivo- de un determinado sujeto social. (Echeverría, 2011, p.567)

Toda proyección y validación de las cualidades de la mercancía (diversas como la sociedad misma) sigue el curso del valor. Solamente cuando el valor de uso es separado realmente de la dualidad co-determinante (su separación de la mercancía como mero sustrato material del valor en general), prosiguiendo un devenir no capitalista, se abren otras posibilidades para su proyección y validación; como categoría, explicaría otra forma de la economía.

Respecto a esto último, ya Marx en su manuscrito de septiembre de 1857, recuperado y analizado por Dussel, mencionaba que "las categorías económicas (...) expresan formas de ser (Daseinsformen), determinaciones de existencia (Existenzbestimmungen) (...)" (Marx citado por Dussel, 1991, p. 56). Siendo así, ¿̇hay otro sentido para el valor de uso? El horizonte del capital no es el único referente. La proyección autónoma de la objetividad de lo material y su realización fuera del ámbito del intercambio (fundado en el acuerdo) son aspectos presentes en la crítica de Bolívar Echeverría. 


\section{La forma simbólica}

Echeverría comienza su argumento preguntándose qué podría constituir otra forma natural ${ }^{3}$ de la reproducción social, diferente de la forma natural que la sociedad moderna capitalista reconoce: la forma natural del progreso técnico, la cual Marx veía con buenos ojos.

Fuertemente influido (...) por la visión del progreso técnico propia del Iluminismo francés que permeaba al Industrialismo ingles de su época, Marx no avanzaría en verdad en el camino de una crítica radical de la forma natural del mundo y de la vida en la época moderna. (Echeverría,1998b, p. 65)

La respuesta la encuentra en el orden cultural del hombre, como pre-condición del proceso técnico y como el "surplus ontológico" (Echeverría,2010b, p. 25) de la reproducción social. En principio, la determinación de la forma del objeto es un asunto social y no del mercado autorregulado. Al plantearlo de esta manera, le devuelve al sujeto el derecho de proyectar la objetividad de lo material y definir un nuevo sentido para las relaciones económicas. Por lo tanto, “en el proceso de reproducción social, la determinación de su figura concreta está (...) entregada a la libertad" (Echeverría, 1998b, p. 166).

Esta libertad debe entenderse a partir de la capacidad que tienen el sujeto de modificar, en un determinado momento, la forma que mejor exprese su socialidad. Dicho de otro modo, al cambiar la sociedad, cambian sus reglas y la forma determinada con la cual se identifica. Los cambios que surgen al interior de la sociedad se reflejan en el proceso de producción-consumo. Según Echeverría (1998b), el compromiso entre el sistema de necesidades y del trabajo se va adecuando a dichos cambios.

Se debe tener en cuenta que en la creación del objeto social no solamente está contemplada su utilidad concreta. Para Echeverría ( $\underline{2010 b} ; \underline{1998 b}$ ), su practicidad (la forma elegida) es también significativa. El objeto práctico es ahora el objeto práctico-significativo; se habla de "producir y consumir significaciones" (Echeverría, 2010b, p. 74).

La forma representativa de la socialidad nunca es definitiva. El no reconocimiento de la forma actual (su significado-significante), en un contexto inter-subjetivo comunicativo, y su consiguiente reconfiguración, es lo que, a juicio de Echeverría (2010b), hace del proceso de reproducción social un proceso de carácter político. Comienza entonces la tensión entre los dos momentos dialécticos de la reproducción social: el momento de la objetivación y el momento de la subjetivación: "Producir y consumir objetos es producir y consumir significaciones. Producir es comunicar (mitteilen), proponer a otro un valor de uso de la naturaleza; consumir es interpretar (auslegen), validar ese valor de uso" (Echeverría, 1998b, p. 182).

${ }^{3}$ La figura representativa de una determinada sociedad.

8

Moisés Martínez Gutiérrez

Revista Economía y Sociedad by Universidad Nacional is licensed under a CreativeCommons Reconocimiento-NoComercial- 
El compromiso, siempre inestable, entre la forma propuesta y su validación lo es respecto al código social, que es, a diferencia del valor económico, el soporte substancial del objeto prácticosignificativo: "Las condiciones requeridas para que tenga lugar esta simbolización (...), esta acción a un tiempo significadora (sobre el contacto) y apropiativa (sobre el referente), se encuentran establecidas en el código" (Echeverría, 1998b, p. 183).

En el proceso de reproducción social no es la omnipresencia del valor sino la del código social lo determinante. El ser humano aparece en dicho proceso (directa y personalmente) para ordenarlo de acuerdo con un proyecto de auto-transformación. Transformación de sí mismo, pero también transformación de otro: el objeto práctico-significativo entra en un proceso abierto de comunicación. Según Echeverría (2010b), pasar de decir algo a sí mismo a decir algo en reciprocidad. Comienza la historia del código social: "Todos los individuos sociales (...) están en un proceso permanente de hacerse a sí mismos, inventando hacer a los otros y dejarse hacer por ellos" (Echeverría,2010b, p.75).

Meta-significar, un acto propiamente político, revela que el compromiso con el código social ya no es vigente, que la socialidad ha cambiado. Esto es fundamental en la explicación que da Echeverría (2010b) sobre la unidad del sistema de capacidades y necesidades del sujeto social, la que permanece en tanto permanezca vigente el compromiso o, como él mismo indica, el sujeto se mueve dialécticamente entre la fidelidad y la desobediencia al compromiso (Echeverría, $\underline{2010 b}$ ). En este sentido tiene que entenderse el proceso de reproducción social.

El ciclo de la reproducción como proceso de vida social (...) es un producir/consumir significaciones, un cifrar/descifrar intenciones transformativas (...) de acuerdo a un código inherente a la estructura tecnológica del propio campo instrumental. (Echeverría,1998b, p. 185)

En el proceso de significar y meta-significar no solamente está en juego el código social. Los principios meta-técnicos van a replantearse. Para empezar, el telos que cumple la técnica moderna tiene su raíz metafísica en la voluntad de poder (Mayz, 1983). En razón de un antropocentrismo y un progresismo que tiende hacia el infinito, el hombre moderno se arroga el derecho de hacer el mundo a su imagen y semejanza, sin fronteras. Al hacerlo, puede disponer de él y superar su finitud: "El fin del hombre, en cuanto actual, es la omnímoda disponibilidad" (García,1986, p.51). Esta disponibilidad siempre es progresiva y supra-natural.

Las ambiciones del hombre moderno capitalista no pueden limitarse a la realización del valor de uso de la mercancía; mas en su conformación este sigue el plan que a todo artefacto moderno le es inherente: "Todo instrumento o máquina moderna (...) lleva (...) el plan propio (...). Haber inventado planes es el equivalente supernatural de inventar esencias" (García, 1986, p. 46). 
La forma concreta que ha nacido con la técnica moderna existe como una invención a disposición del capitalista, pero la técnica moderna radicaliza esta situación. La progresiva efectividad de la técnica moderna (que ha superado ya la propuesta espontánea de lo útil natural) supone un modo de la disponibilidad. Ahora, indica Heidegger (1997), la naturaleza es puesta en el camino de un develar provocado, una liberación de energía conducida por el hombre, distinguiéndose, por lo tanto, de la poiesis (un develar en libertad).

Esta técnica moderna, coadyuvante en la superación de la finitud del ser humano, es el soporte del modo de producción capitalista. Dicho en otros términos, el plan técnico del capitalista sigue su raíz metafísica: la voluntad de poder. En realidad, no hay una efectividad única de la técnica. Mayz (1983) afirma que todo a priori técnico está condicionado "epocalmente", que no hay una efectividad única de la técnica. Esta idea es compartida por Echeverría (1998b), para quien la efectividad de la técnica permanece abierta (la configuración libre del objeto social). Ello supondría la intervención del sujeto en la modalidad del objeto social.

Usar el campo instrumental consiste por un lado en obedecer y por otro en rebelarse al proyecto de objetividad que él trae consigo en su composición técnica; es intervenir en la historia de la producción/consumo como historia de la relación sujeto-objeto. (Echeverría,1998b, p. 186)

Al cambiar la socialidad, cambia el código social y todo principio meta-técnico del hombre. Así pues, el planteamiento de Echeverría no está centrado en el objeto sino en el sujeto de la reproducción social. Un volver a la sujetidad, a la auto-proyección, con independencia de las leyes del mercado capitalista.

\section{Conclusión}

El giro efectuado por Echeverría hacia el valor de uso tiene una razón: comenzar la problematización de la forma natural de la reproducción social, una forma que, a diferencia de la mercancía, tiene un estrato de objetividad práctica y simbólica. ¿Por qué? No solamente el valor, su reproducción y enajenación son determinantes en la vida económica del ser humano, todo proceso de reproducción social es un proceso comunicativo-significativo. En este sentido, la forma representativa de una sociedad y su reconfiguración sémica permanente es fundamental. Por lo tanto, en el análisis del valor de uso se debe considerar su proceso interno de significación y meta-significación, así como su proceso externo de convalidación-negación, a la luz de los cambios sociales y sin perder de vista su estado actual: subordinado al valor y al telos de la técnica moderna.

10

Moisés Martínez Gutiérrez

Revista Economía y Sociedad by Universidad Nacional is licensed under a CreativeCommons Reconocimiento-NoComercial- 


\section{Referencias}

Dussel, E. (1991). La producción teórica de Marx, un comentario a los Grundrisse. Recuperado de http://biblioteca.clacso.edu.ar/gsdl/cgi-bin/library.cgi?e=d-11000-00---off-0otros--00-1----010-0---0---0direct-10---4-------0-0|--11-es-Zz-1---20-about---00-3-1-00-0--4----0-0-01-00OutfZz-8-00\&a=d\&cl=CL3.4\&d=D5665.1

Echeverría, B. (1998a). La contradicción del valor y el valor de uso en El Capital, de Karl Marx. Recuperado de http://www.flacsoandes.edu.ec/libros/124995-opac

Echeverría, B. (1998b). Valor de uso y utopía. Recuperado de https://books.google.co.cr/books/about/Valor de uso y utop\%C3\%ADa.html?id=jPs7A7w dYHcC\&redir esc=y

Echeverría, B. (2010a). Modernidad y blanquitud. Recuperado de http://www.edicionesera.com.mx/sociedad-y-politica/modernidad-y-blanquitud-info

Echeverría, B. (2010b). Definición de la cultura Recuperado de https://www.elfondoenlinea.com/Detalle.aspx?ctit=014568R

Echeverría, B. (2011). Crítica de la modernidad capitalista. Recuperado de https://www.vicepresidencia.gob.bo/IMG/pdf/bolivar echeverria.pdf

García, J. (1986). Elogio de la técnica. Recuperado de http://www.anthroposeditorial.com/DETALLE/ELOGIO-DE-LA-TECNICA-PCPU-025

Heidegger, M. (1997). Filosofía, ciencia y técnica. Recuperado de https://www.casadellibro.com/libro-filosofia-ciencia-y-tecnica-4ed/9789561113114/898279

Hinkelammert, F. (2007). Hacia una crítica de la razón mítica. Recuperado de http://www.pensamientocritico.info/index.php/libros/libros-de-franzhinkelammert?download=1:critica-de-la-razon-mitica-critica-franz-hinkelammert

Hinkelammert, F. (2012). Lo indispensable es inútil hacia una espiritualidad de la liberación. Recuperado de http://www.pensamientocritico.info/index.php/libros/libros-de-franzhinkelammert/espanol?download=27:Lo\%20indispensable\%20es\%20in\%C3\%BAtil:\%2OHacia \%20una\%20espiritualidad\%20de\%20la\%20liberaci\%C3\%B3n-lo-indispensable-es-inutilfranz-hinkelammert 
Hinkelammert, F. y Mora, H. (2013). Hacia una economía para la vida. Recuperado de http://catalogo.koha.umich.mx/cgi-bin/koha/opac-detail.pl?biblionumber=94668.

Marx, K. (1975). Fundamentos de la crítica de la economía política. La Habana: Editorial de Ciencias Sociales.

Marx, K. (1976). Contribución a la crítica de la economía política. Recuperado de http://librunam.dgbiblio.unam.mx:8991/F/YGU5BTQ2SUVQK1NXXL9IEY2YU15QATLJNDYV8 1FQTX6NGQ43SR-43460?func=full-setset\&set number $=031583 \&$ set entry $=000012 \&$ format $=999$

Marx, K. (1984). El Capital. México: Fondo de Cultura Económica.

Mayz, E. (1983). Ratio Technica. Recuperado de https://www.casadellibro.com/libro-ratiotechnica/9789999119207/488067

Picó, J. (1999). Cultura y modernidad. Recuperado de https://books.google.es/books/about/Cultura y modernidad.html?hl=es\&id=-6dfmcGlpy4C

Polanyi, K. (2009). La gran transformación. Recuperado de http://librunam.dgbiblio.unam.mx:8991/F/YGU5BTQ2SUVQK1NXXL9IEY2YU15QATLJNDYV8 1FQTX6NGQ43SR-70467?func=full-setset\&set number $=032993 \&$ set entry $=000010 \&$ format $=999$

Rubel, M. (2003). Marx sin mito. Barcelona: OCTAEDRO. Recuperado de https://www.casadellibro.com/libro-marx-sin-mito/9788480635639/881725

12 